\title{
Endogenous Parathyroid Hormone Promotes Fracture Healing by Increasing Expression of BMPR2 through cAMP/PKA/ CREB Pathway in Mice
}

\author{
Wei Zhou ${ }^{a}$ Lipeng Yu Jin Fan ${ }^{a}$ Bowen Wan ${ }^{a}$ Tao Jiang ${ }^{a}$ Jian Yin ${ }^{a}$ Yao Huang ${ }^{a}$ \\ Qingqing $\mathrm{Li}^{\mathrm{a}}$ Guoyong Yin ${ }^{\mathrm{a}}$ Zhaoxing $\mathrm{Hu}^{\mathrm{a}, \mathrm{b}}$ \\ aDepartment of Orthopaedics, The First Affiliated Hospital of Nanjing Medical University, Nanjing, \\ bDepartment of Orthopaedics, The Second Affiliated Hospital of Nanjing Medical University, Nanjing, \\ China
}

\section{Key Words}

PTH・ Fracture healing • BMPR2 • CAMP/PKA/CREB

\begin{abstract}
Background/Aims: Endogenous parathyroid hormone (PTH) plays an important role in fracture healing. This study investigated whether endogenous PTH regulates fracture healing by bone morphogenetic protein (BMP) and/or the transforming growth factor- $\beta$ (TGF- $\beta$ ) signaling pathway. Methods: Eight-week-old wild-type (WT) and PTH-knockout (PTH KO) male mice were selected, and models of open right-femoral fracture were constructed. Fracture healing and callus characteristics of mice in the two groups were compared by X-ray, microcomputed tomography, histological, and immunohistochemical examinations. Bone marrow mesenchymal stem cells (BMMSCs) of 8-week-old WT and PTHKO male mice were obtained and induced into osteoblasts and chondrocytes. Results: We found that expression levels of Runt-related transcription factor (RUNX2), bone morphogenetic protein-receptor-type II (BMPR2), phosphorylated Smad 1/5/8, and phosphorylated cyclic adenosine monophosphateresponsive element binding protein (CREB) in the callus of PTHKO mice were significantly decreased, whereas no significant difference in expression of SOX9, TGF- $\beta$ R2, or pSMAD2/3 was observed between PTHKO and WT mice. Additionally, the activity of osteoblast alkaline phosphatase was low at 7 days post-induction, and was upregulated by addition of PTH or dibutyryl cyclic adenosine monophosphate (dbcAMP) to the cell culture. Furthermore, H89 (protein kinase A inhibitor)eliminated the simulating effects of PTH and dbcAMP, and a low concentration of cyclic adenosine monophosphate (CAMP) was observed in PTHKO mouse BMMSCs. Conclusion: These results suggested that endogenous PTH enhanced BMPR2 expression by a CAMP/PKA/CREB pathway in osteoblasts, and increased RUNX2 expression through transduction of the BMP/pSMAD1/5/8 signaling pathway.

W. Zhou and L. Yu contributed to this work equally

Guoyong Yin and Zhaoxing Hu

Department of Orthopaedics, No. 300, Guangzhou Road, Nanjing, 210029 Jiangsu Provinc, (The People's Republic of China)

E-Mail guoyong_yin@sina.com / hulaker1@sohu.com
\end{abstract}

KARGER 


\section{Cellular Physiology Cell Physiol Biochem 2017;42:551-563 \\ \begin{tabular}{l|l} 
DOI: 10.1159/000477605 & and Biochemistry \\
Published online: June 05, 2017 & $\begin{array}{l}\text { O 2017 The Author(s). Published by S. Karger AG, Basel } \\
\text { www.karger.com/cpb }\end{array}$
\end{tabular} \\ Zhou et al.: Endogenous PTH Enhanced BMPR2 by cAMP/PKA/CREB Pathway}

\section{Introduction}

There are two major modes of bone formation during fracture healing: intramembranous ossification and endochondral ossification $[1,2]$, with the latter always occurring closest to the fracture site. During the process, local bone marrow mesenchymal stem cells (BMMSC) gather under hypoxic conditions and differentiate into chondrocytes, which secrete cartilage matrix. As chondrocytes mature, degenerate, and ultimately undergo apoptosis, osteoblasts enter the cartilage matrix where they gradually form woven bones. Intramembranous ossification primarily takes place at both ends of bony callus, and new bones are formed by proliferation of periosteal osteoblasts [2,3]. The process of fracture healing is completed as callus is remodeled, with functions of both osteoclasts and osteoblasts according to appropriate mechanical requirements $[4,5]$.

The transforming growth factor $\beta$ (TGF- $\beta$ ) superfamily, which includes TGF- $\beta$ and bone morphogenetic protein (BMP), plays an important role during fracture healing [6]. While TGF- $\beta$ is crucial to the growth and differentiation of various types of cells, including chondrocytes, and is necessary for the differentiation of mesenchymal stem cell (MSC) into chondrocyte [7], BMP can promote osteogenic differentiation and MSC maturation, and is the only growth factor in the TGF- $\beta$ superfamily capable of inducing bone formation independent of other factors $[8,9]$. In the TGF- $\beta$ /BMP signaling pathway, ligands bind to type II transmembrane serine/threonine kinase receptors to form heteromeric complexes. Type II receptors phosphorylate and activate type I receptors [10], which activate specific receptor-activated Smad proteins (R-Smad) by phosphorylation. R-Smads form complexes with Smad4 and translocate into the nucleus, where they cooperatively regulate the transcription of target genes with other transcription factors [11]. Transcription factors regulating osteogenesis in the TGF- $\beta$ /BMP pathway include positive regulatory factors, such as RUNX2 and Osterix, and negative regulatory factors, such as CIZ and AJ18 [8]. During the complex differentiation process of MSCs into chondrocytes and osteoblasts, sex determinig region of Y choromosome (SRY)-related high-mobility-group box 9 (SOX9) promotes chondrogenic differentiation of MSCs, and RUNX2 controls their osteoblastic differentiation. RUNX2 also stimulates chondrocyte maturation and hypertrophy, whereas SOX9 inhibits this process [12].

Parathyroid hormone (PTH) is a polypeptide hormone secreted by the parathyroid glands, andregulates calcium and phosphorus homeostasis in the body [13]. Intermittent PTH application can improve bone strength and increase bone density $[14,15]$. Teriparatide [recombinant human PTH (1-34)] has been used to treat osteoporosis in postmenopausal women [16]. Additionally, animal experiments have confirmed that intermittent PTH (1-34) administration can accelerate fracture healing by stimulating endochondral bone formation and promoting osteoblastic differentiation of MSCs [17-19]. However, the mechanism behind PTH stimulation of fracture healing remains unclear. It is generally believed that PTH functions are primarily mediated through binding to G-protein-coupled PTH receptor (PTHR1) and activating G-mediated adenylyl cyclase (AC), which generates the secondmessenger cAMP. PKA, activated by cAMP, regulates the expression of proteins involved in the cell cycle, related kinase inhibitors, and RUNX2 [20]. PTH directly stimulates osteoblast proliferation through this pathway, leading to increased cell numbers and reduced apoptosis. However, PTH also affects synthesis of osteogenic growth factors and cytokines, such as insulin-like growth factor 1 , fibroblast growth factor 2 , and Wnts, as well as their antagonists or proteolytic activators, thereby indirectly promoting differentiation and proliferation of osteoblasts and their precursors [17].

In this study, we investigated the effects of PTH deletion on the BMP and TGF- $\beta$ signaling pathways during fracture healing using a PTH KO mouse model. Our results indicated that endogenous PTH enhanced BMPR2 expression in osteoblasts through the cAMP/PKA/CREB pathway, and promoted RUNX2 expression by increasing signal transduction inthe BMP/ pSMAD1/5/8 pathway. These findings suggest that PTH might affect osteoblast functions at the fracture site through these pathways and ultimately promoted fracture healing. 


\section{Cellular Physiology Cell Physiol Biochem 2017;42:551-563 \begin{tabular}{l|l} 
DOI: 10.1159/000477605 & Ond Biochemistry 2017 The Author(s). Published by S. Karger AG, Basel \\
Published online: June 05, 2017 & $\begin{array}{l}\text { www.karger.com/cpb } \\
\text { and }\end{array}$
\end{tabular} Zhou et al.: Endogenous PTH Enhanced BMPR2 by cAMP/PKA/CREB Pathway}

\section{Materials and Methods}

Animals

Pairs of C57BL/6J male and female mice heterozygous for the PTH allele (PTH +/-) were randomly selected and mated to produce PTH wild-type (WT; PTH +/+) and PTH homozygous (PTH -/-) offspring. The breeder mice were donated by Dr. Ren (Department of Orthopaedics, The First Affiliated Hospital of Nanjing Medical University, Nanjing, China).Mice were housed in the specific pathogen-flee (SPF) animal center in Nanjing Medical University. Eight-week-old homozygous PTH KO male mice and wild-type (WT) littermates were randomly selected for this study. All animal experiments were approved of by and carried out in accordance to the guidelines of Ethical Committee for Laboratory Experimentation (ECD) of Nanjing Medical University. All efforts were made to minimize animal suffering.

Identification of animal genotypes

DNA fragments from mouse tails were extracted by phenol-chloroform-isoamyl alcohol, and the genotypes of mice were identified by polymerase chain reaction (PCR) amplification. All primers were synthesized by Invitrogen (Shanghai Invitrogen, Shanghai, China). Primer sequences were as follows: forward primer for the PTH gene, 5'- GATGTGTGCAAACACCGTGGCTAA -3'; reverse primer, 5'- TCCAAAGTTTCATTACAGTAGAAG -3'; forward primer for the neomycin-resistance gene (Neo), 5'-TCTTGATTCCCACTTTGTGGTTCTA-3'; reverse primer, 5'-TCCAAAGTTTCATTACAGTAGAAG-3'. PCRs were performed using the following cycle conditions: initial denaturation at $94^{\circ} \mathrm{C}$ for $4 \mathrm{~min}$; 30 cycles of denaturation at $94^{\circ} \mathrm{C}$ for $45 \mathrm{~s}$, annealing at $55^{\circ} \mathrm{C}$ for $45 \mathrm{~s}$, and extension at $72^{\circ} \mathrm{C}$ for $45 \mathrm{~s}$, followed by cooling to $4^{\circ} \mathrm{C}$. The expected length of the amplified PTH and Neo gene fragments were 451 and $276 \mathrm{bp}$, respectively. Mice with only a $451 \mathrm{bp}$ amplification product for the PTH gene, a $276 \mathrm{bp}$ amplification product for the Neo gene, or both were identified as WT, homozygous PTH KO, or heterozygous mice, respectively.

\section{Construction of the murine fracture model}

Eight-week-old PTH KO male mice and WT littermates were sedated with isoflurane (Forene ${ }^{\circledR}$ ) and anesthetized with an intraperitoneal injection of $1 \%$ sodium pentobarbital (Sigma Aldrich, St. Louis, MO, USA) (1 uL/g). Skin tissue around the right leg was disinfected with povidone-iodine after shaving hair, and an open fracture model was established according to the procedure described previously [21, 22]. Briefly, an 8-mm-long incision was made between the right knee and the hip in a sterile environment, the right femoral shaft was exposed by blunt dissection between the vastus lateralis muscle and the fascia, and the femoral shaft was cut in the middle with a special pair of scissors made for ingrown nails (Guangzhou FANGZE, Guangzhou, China). Brocken ends of the fracture were repositioned by inserting a syringe needle $(0.45-\mathrm{mm}$ diameter) into the bone marrow cavity of the right femur as a retrograde intramedullary fixation device. The needle was inserted until it penetrated the proximal femur, and then the end of the needle was cut and the incision was then sutured. No mice were infected or died.

The mice $(n=5)$ were selected at 7,14 , and 21 days after the surgery, and sacrificed by cervical dislocation. Right femurs were isolated and soft tissues carefully removed. The femurs were fixed in $2 \%$ paraformaldehyde, $75 \mathrm{mM}$ lysine, and $10 \mathrm{mM}$ sodium iodide (PLP) for $24 \mathrm{~h}$ and stored in phosphatebuffered saline (PBS). The femurs were examined by X-ray and micro-computed tomography (CT). The specimens were then decalcified in ethylenediaminetetraacetic acid at $4^{\circ} \mathrm{C}$ for 15 to 20 days, dehydrated, embedded in paraffin, and cut into $5 \mu \mathrm{m}$ serial sections, which were subject to hematoxylin and eosin (H\&E) and immunohistochemical staining.

\section{$X$-ray and micro-CT examination}

Undecalcified femoral specimens were examined using a Faxitron model 805 radiographic inspection system (Faxitron Contact, Faxitron, Germany; 22-kV voltage and 4-min exposure time). These specimens were also scanned by the SkyScan 1176 (Antwerp, Belgium) and analyzed by its three-dimensional imaging software using the following conditions: $40 \mathrm{kV}$ accelerating voltage, $98 \mu \mathrm{m}$ beam diameter, $0.9^{\circ}$ rotation space, $18.2 \mu \mathrm{m}$ resolution, and minimum contrast threshold.

\section{Cell culture}

Eight-week-old PTHKO and WT mice were anesthetized with ether, sacrificed by cervical dislocation, and immersed in $75 \%(\mathrm{v} / \mathrm{v})$ ethanol for 3-5 min. BMMSCs were obtained from the marrow cavity of the 


\section{Cellular Physiology Cell Physiol Biochem 2017;42:551-563 \begin{tabular}{ll|l} 
DOI: 10.1159/000477605 & $\begin{array}{l}\text { O 2017 The Author(s). Published by S. Karger AG, Basel } \\
\text { www.karger.com/cpb }\end{array}$
\end{tabular} \\ Zhou et al.: Endogenous PTH Enhanced BMPR2 by cAMP/PKA/CREB Pathway}

femur and tibia. Single-cell suspensions were prepared and seeded into 10 -cm-diameter dishes $(5 \mathrm{~mL}$ per dish) with complete Dulbecco's modified Eagle's medium (DMEM, Sigma Aldrich, St. Louis, MO, USA) medium [ $10 \%$ fetal bovine serum (Gibco, Grand Island, NY, USA), 2 mmol/L glutamine (Sigma Aldrich), 100 $\mathrm{U} / \mathrm{mL}$ penicillin and $100 \mathrm{mg} / \mathrm{L}$ streptomycin (penicillin-streptomycin, Sigma Aldrich)]. Cells were incubated at $37^{\circ} \mathrm{C}$ with $5 \%(\mathrm{v} / \mathrm{v}) \mathrm{CO}_{2}$. Half of the medium was replaced with fresh complete medium after $72 \mathrm{~h}$, and culture medium was then changed every $48 \mathrm{~h}$. Cells $>80 \%$ confluence were digested with $2.5 \mathrm{~g} / \mathrm{L}$ trypsin, collected by centrifugation $\left(37^{\circ} \mathrm{C}, 1000 \mathrm{~g}, 5 \mathrm{~min}\right)$, resuspended, and subcultured in fresh medium at a 1:2 ratio.

MSCs were induced into chondrocytes as follows: cells $>80 \%$ confluence were digested with $2.5 \mathrm{~g} / \mathrm{L}$ trypsin, and the cell suspension was transferred to a $15-\mathrm{mL}$ centrifuge tube. The tube was centrifuged $\left(37^{\circ} \mathrm{C}, 1000 \mathrm{~g}, 5 \mathrm{~min}\right)$, the supernatant discarded, and $3 \mathrm{~mL}$ chondrogenic induction medium (F-12 medium containing $10 \% \mathrm{FBS}, 10 \mathrm{ng} / \mathrm{mL}$ TGF- $\beta, 10 \mathrm{nmol} / \mathrm{L}$ dexamethasone, and $50 \mu \mathrm{g} / \mathrm{mL}$ ascorbic acid) added. Cell micelles were cultured in the centrifuge tube, with the medium changed every $48 \mathrm{~h}$. The medium was discarded after 12 days, and samples were fixed in PLP for $24 \mathrm{~h}$, dehydrated, embedded in paraffin, cut into $5 \mu \mathrm{m}$ serial sections, and subject to H\&E and immunohistochemical staining.

MSCs were induced into osteoblasts according to the following protocol: cells $>80 \%$ confluence were starved in serum-free DMEM medium for $6 \mathrm{~h}$, then $5 \mathrm{~mL}$ osteogenic induction medium (DMEM medium containing 10\% FBS, $10 \mathrm{nmol} / \mathrm{L}$ dexamethasone, $10 \mathrm{mmol} / \mathrm{L} \beta$-glycerophosphate, and $50 \mu \mathrm{g} / \mathrm{mL}$ ascorbic acid) was added, and the medium was changed every $48 \mathrm{~h}$. cells were cultured with or without stimulation with PTH(0.1 $\mu$ M, Sigma Aldrich, St. Louis, MO, USA), dibutyryl-cAMP (dbcAMP; $100 \mu$ M),or PKA inhibitor H89 $(100 \mu \mathrm{M})$. Total cellular proteins were extracted4 days after induction and analyzed by western blot. Alkaline phosphatase (ALP) staining and ALP-activity tests were performed 7 days after induction.

\section{Western blot analysis}

Proteins were extracted from cells $(n=4)$ using radioimmunoprecipitation assay lysis buffer (Beyotime Biotechnology, Shanghai, China) and quantified by the Bradford method. After sodium dodecyl sulfate polyacrylamide gel electrophoresis, protein samples were transferred to polyvinylidene fluoride membranes (Millipore, Billerica, MA, USA),which were incubated with the primary antibody overnight at $4^{\circ} \mathrm{C}$, and then incubated with horseradish peroxidase-conjugated secondary antibody (Santa Cruz Biotechnology, Dallas, TX, USA) for $2 \mathrm{~h}$ at $37^{\circ} \mathrm{C}$.Electrogenerated chemiluminescence(Beyotime Biotechnology) was performed, and protein bands were analyzed using Quantity One software (Bio-Rad Laboratories, Hercules, CA, USA).

\section{H\&E and immunohistochemical staining}

Paraffin sections were conventionally dewaxed, hydrated, and stained with hematoxylin (Sigma Aldrich). Sections were differentiated in $1 \%$ acid-alcohol, rinsed with running water, and stained with eosin (Sigma). Sections were then dehydrated, cleared, and mounted with neutral gum.

Since the activities of all signal pathways in the callus of both PTHKO and WT mice were significantly reduced, and off-chip occurred easily at 21-days post-fracture, sections prepared at 7- and 14-days postfracture were used instead for immunohistochemical staining. Paraffin sections were conventionally baked, dewaxed, hydrated, hot fixed, and treated with 5\% hydrogen peroxide for 20 min, followed by incubating with $5 \%$ homologous serum for 1 h. Serum was removed, and sections were incubated with specific primary antibodies [rabbit polyclonal antibody to typesI and II collagen, TGF- $\beta$ R2, RUNX2, SOX9, and phosphorylated CREB (pCREB); mouse monoclonal antibody to BMPR2 (Abcam, Cambridge, MA, USA); and rabbit monoclonal antibody to pSMAD2/3 and pSMAD1/5/8 (Santa Cruz Biotechnology)] at a 1:100 dilution in PBS/bovine serum albumin overnight at $4^{\circ} \mathrm{C}$.The primary antibody was removed, and sections were incubated with goat anti-rabbit IgG (Sigma Aldrich) at a 1:200 dilution at $37^{\circ} \mathrm{C}$ for $1 \mathrm{~h}$. Sections were incubated with a 3,3'-diaminobenzidine solution (Boster Biotechnology, Wuhan, China) in the dark at room temperature for $\sim 4-5 \mathrm{~min}$, counterstained with hematoxylin for $2.5 \mathrm{~min}$, dehydrated, cleared, and mounted with neutral gum. Stained sections were observed bylight microscopy, and digital photographs were taken.

\section{Detection of ALP activity}

The activity of osteoblast differentiation marker ALP reflected the effects of PTHKO on the osteogenetic process of MSCs. BMMSCs from PTHKO and WT mice were inoculated in 24-well plates at a concentration of $1 \times 10^{5}$ cells/well $(\mathrm{n}=4)$. Cells at $100 \%$ confluence were starved in serum-free DMEM medium for $6 \mathrm{~h}$, 


\section{Cellular Physiology Cell Physiol Biochem 2017;42:551-563 \begin{tabular}{l|l} 
DOI: 10.1159/000477605 & $\begin{array}{l}\text { O 2017 The Author(s). Published by S. Karger AG, Basel } \\
\text { www.karger.com/cpb }\end{array}$
\end{tabular} \\ Zhou et al.: Endogenous PTH Enhanced BMPR2 by cAMP/PKA/CREB Pathway}

cells in the H89 group were pretreated with $100 \mu \mathrm{M}$ PKA inhibitor H89 for $1 \mathrm{~h}$. The H89 was discarded, and cells were incubated in osteogenic induction medium containing $0.1 \mu \mathrm{M}$ PTH (Sigma Aldrich) and dbcAMP for 7 days. The activity of ALP in cells 7 days after stimulation was measured using an ALP-detection kit (Beyotime Biotechnology) according to manufacturer instructions.

\section{ALP staining}

MSCs were seeded into six-well plates $(n=4)$, and cells $>80 \%$ confluence were starved in serum-free DMEM medium for $6 \mathrm{~h}$. Cells were incubated in osteogenic induction medium and fixed with PLP at 7-days post-induction. ALP staining was performed using an ALP-staining kit (Jiancheng Bioengineering, Jiangsu, China) following standard procedures. Samples were counterstained with hematoxylin, rinsed three times with PBS, observed by light microscopy, and digital photographs obtained.

\section{Detection of intracellular cAMP concentration}

BMMSCs from PTHKO and WT mice were inoculated in 12-well plates $(n=3)$. Cells at $100 \%$ confluence were starved in serum-free DMEM medium for $1 \mathrm{~h}$, and serum-free medium was replaced by complete

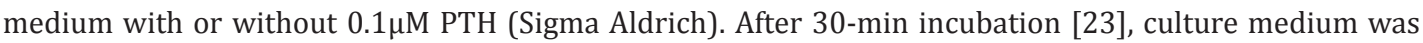
discarded and intracellular cAMP levels in the cell lysate were measured using the cAMP parameter assay kit (R\&D Systems, Minneapolis, MN, USA) following the standard protocol.

\section{Statistical analysis}

All images from H\&E and immunohistochemical stains were obtained using a digital microscope camera (Leica, Wetzlar, Germany), and quantitatively analyzed using Northern Eclipse software(Empix Imaging, Mississauga, $\mathrm{ON}$, Canada). Differences among groups were analyzed by single-factor analysis of variance using SPSS 20.0 software (IBM Corp., Chicago, IL, USA). A p $<0.05$ was considered statistically significant.

\section{Results}

PTH knockout caused delayed fracture healing and reduced bone density in fractured femurs

X-ray examination showed that all mouse fracture models contained simple transverse fractures. PTHKO mice displayed slower callus formation and longer recovery periods after fracture compared to WT mice (Fig 1A). Bone mineral densities of the callus were measured using micro-CT, showing that the BMD of the PTHKO group at 14- and 21-days

Fig. 1. Endogenous PTH deficiency impairs fracture healing and bone mineral density of callusin fractured femurs. (A): Fractures were examined using X-ray and micro CT at 7, 14 and 21 days after surgery, respectively, and three-dimensional reconstruction of target surface was performed. (B): Comparison of BMD of callus in PTHKO and WT mice at each time point. Each value is the mean $\pm \mathrm{SD}$ of determinations in fiveanimals from each group. ( $*$ P $<0.05 \mathrm{KO}$ mice compared with WT mice).

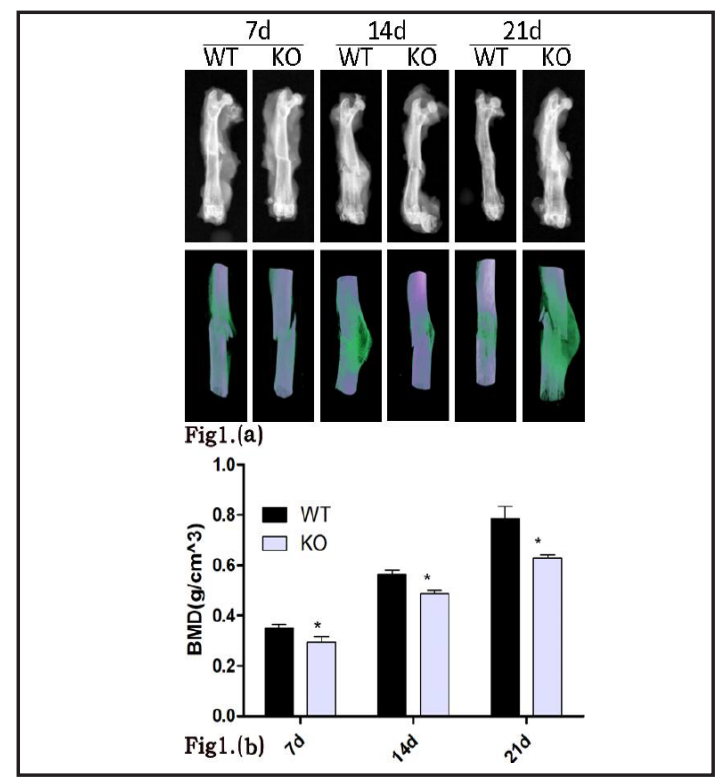


Fig. 2. Endogenous PTH promoted the differentiation of BMMSCs into osteoblasts, but had no effect on the differentiation into chondrocytes. (A): Microscopy images of ALP staining of BMMSCs at 7 days after osteogenic induction (200x). The black arrow refers to the mature osteoblast, and the white arrow refers to pre-osteoblastic or BMMSC. (B): Comparison of the number of mature osteoblasts in ALP staining between PTHKO and WT group. (C): H\&E staining and immunohistochemical staining of Col II of paraffin sections at 12 days after chondrogenic induction of BMMSCs $(200 \times)$. (D): Comparison of Col II staining intensity of cell-micelles in PTHKO and WT group, and there have no significant difference. ${ }^{*}$ : P $<0.05$ KO mice compared with WT mice).

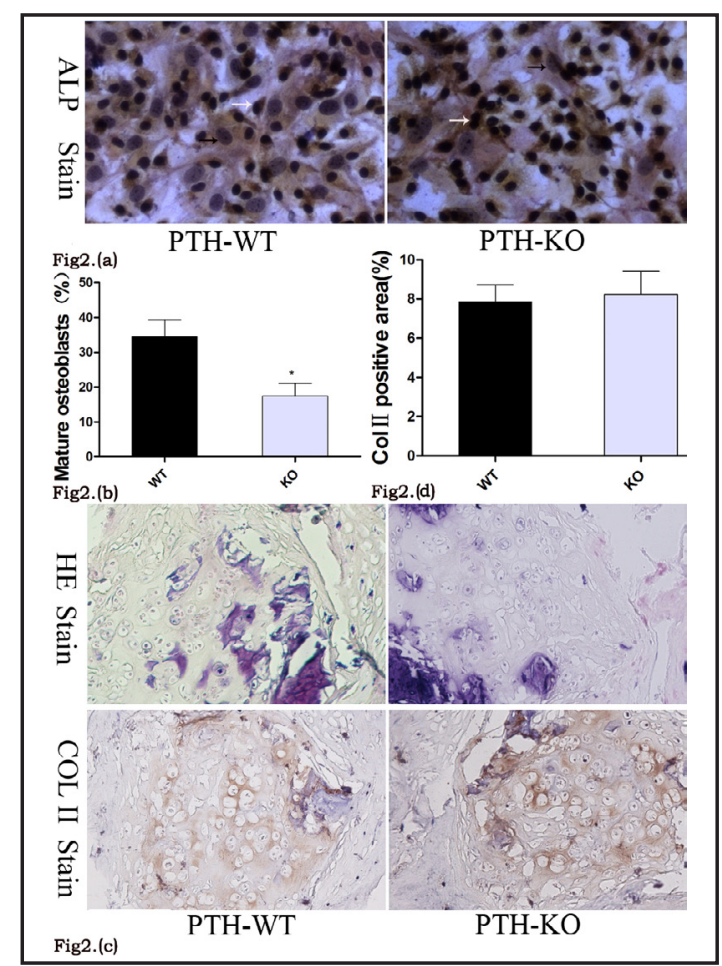

post-surgery was $0.478 \pm 0.014$ and $0.630 \pm 0.012 \mathrm{~g} / \mathrm{cm}^{3}(\mathrm{p}<0.001)$, respectively, whereas the corresponding BMD values for WT mice were $0.538 \pm 0.040$ and $0.786 \pm 0.050 \mathrm{~g} / \mathrm{cm}^{3}$ (Figure 1B), respectively, suggesting reduced bone density and decreased bone mass of the callus during fracture healing in PTHKO mice (at 14and 21days). Callus formation in PTHKO mice was significantly slower than that in WT mice at 7- and 14-days post-fracture, and callus remodeling had already taken place in WT mice at 21-days post-fracture, whereas the giant callus in PTHKO mice was still in the maturation phase of lamellar bones (Fig .1).

Assessment of the effects of PTH knockout on BMMSC differentiation into chondrocytes and osteoblasts

ALP staining of BMMSCs at 7 days after osteogenic induction demonstrated that the ratio of mature osteoblasts in the WT group was significantly higher than that observed in the PTH KO group ( $\mathrm{p}<0.05$ ) (Fig. 2A and B). BMMSCs formed micelles at the bottom of a centrifuge tube after centrifugation, resulting in localized hypoxia inside the micelles. Micelles were induced into chondrocytes and subjected to H\&E and immunohistochemical staining at 12-days post-induction. No significant differences in the number and functions of BMMSC-derived chondrocytes were observed between the PTHKO and WT groups ( $p>0.05$ ), and no significant difference in staining intensity of Col II was identified (Fig. 2C and D).

Assessment of the effects of PTH knockout on chondrocyte and osteoblast function

Immunohistochemical results revealed significantly reduced expression of both Col I and RUNX2 in the callus of PTHKO mice as compared to WT mice $(p<0.05)$, while no significant difference was observed between the expression of SOX9 in the two groups ( $p>$ 0.05) (Fig. 3). The percentage of Col II staining area was approximately the same in the two groups at 7-days post-fracture. This value in the WT group dropped significantly at 14-days post-fracture, while that of the PTHKO group exhibited no significant decrease (Fig. 3A and B), indicating delayed chondrocyte death and defective endochondral ossification.

Assessment of the effects of PTH knockout on the TGF- $\beta$-signaling pathway

Immunohistochemical staining was performed on callus sections to investigate the effects of PTH knockout on cellular signal transduction within the TGF- $\beta$-pathway. No 


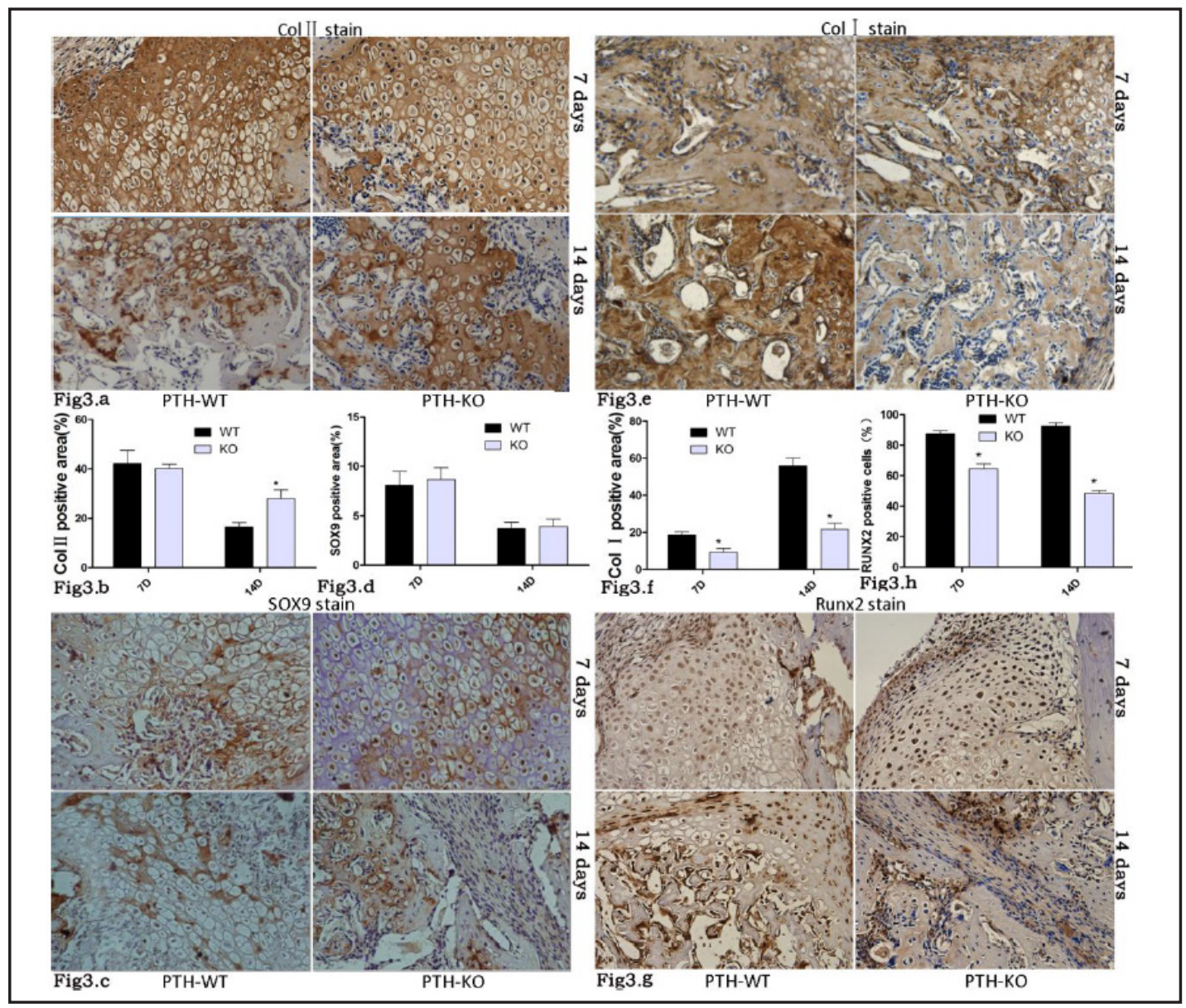

Fig. 3. Endogenous PTH deficiency impaired the function of osteoblasts in the callus ,but had no effect in chondrocytes. (A): Immunohistochemical staining of Col II in the callus of both PTHKO and WT mice at 7 and 14 days after fracture (200x). (B): Comparison of immunohistochemical staining intensity of Col II in callus. (C) : Immunohistochemical staining of SOX9 in the callus of both PTHKO and WT mice at 7 and 14 days after fracture (200×). (D): Comparison of immunohistochemical staining intensity of SOX9. (E) : Immunohistochemical staining of Col I in the callus of both PTHKO and WT mice at 7 and 14 days after fracture (200x). (F): Comparison of immunohistochemical staining intensity of Col I. (G) : Immunohistochemical staining of Runx2 in the callus of both PTHKO and WT mice at 7 and 14 days after fracture $(200 \times)$. $(\mathrm{H})$ : Comparison of immunohistochemical staining intensity of Runx2. (*: P <0.05 KO mice compared with WT mice).

significant differences in expression of TGF- $\beta$ R2 and pSMAD2/3 in the callus were identified between PTHKO and WT mice (p>0.05) (Fig. 4).

PTH deletion reduced intracellular signal transduction in theBMP pathway

The effects of PTH deletion on the BMP signaling pathway during fracture healing was examined, with immunohistochemical staining of the callus demonstrating that expression of BMPR2 and pSMAD1/5/8 in the callus of PTHKO mice was significantly lower as compared to that observed in WT mice $(\mathrm{p}<0.05)$ (Fig. 5A-D).

PTH deletion reduced intracellular CAMP levels and decreased CREB expression

Immunohistochemical results revealed a significant reduction in the expression of pCREB (Fig. 6A and B). Similarly, the intracellular concentration of the second messenger CAMP in the PTH signaling pathway was measured to investigate the regulatory mechanism of PTH on the BMP signaling pathway. The results indicated that intracellular cAMP levels in

\section{KARGER}




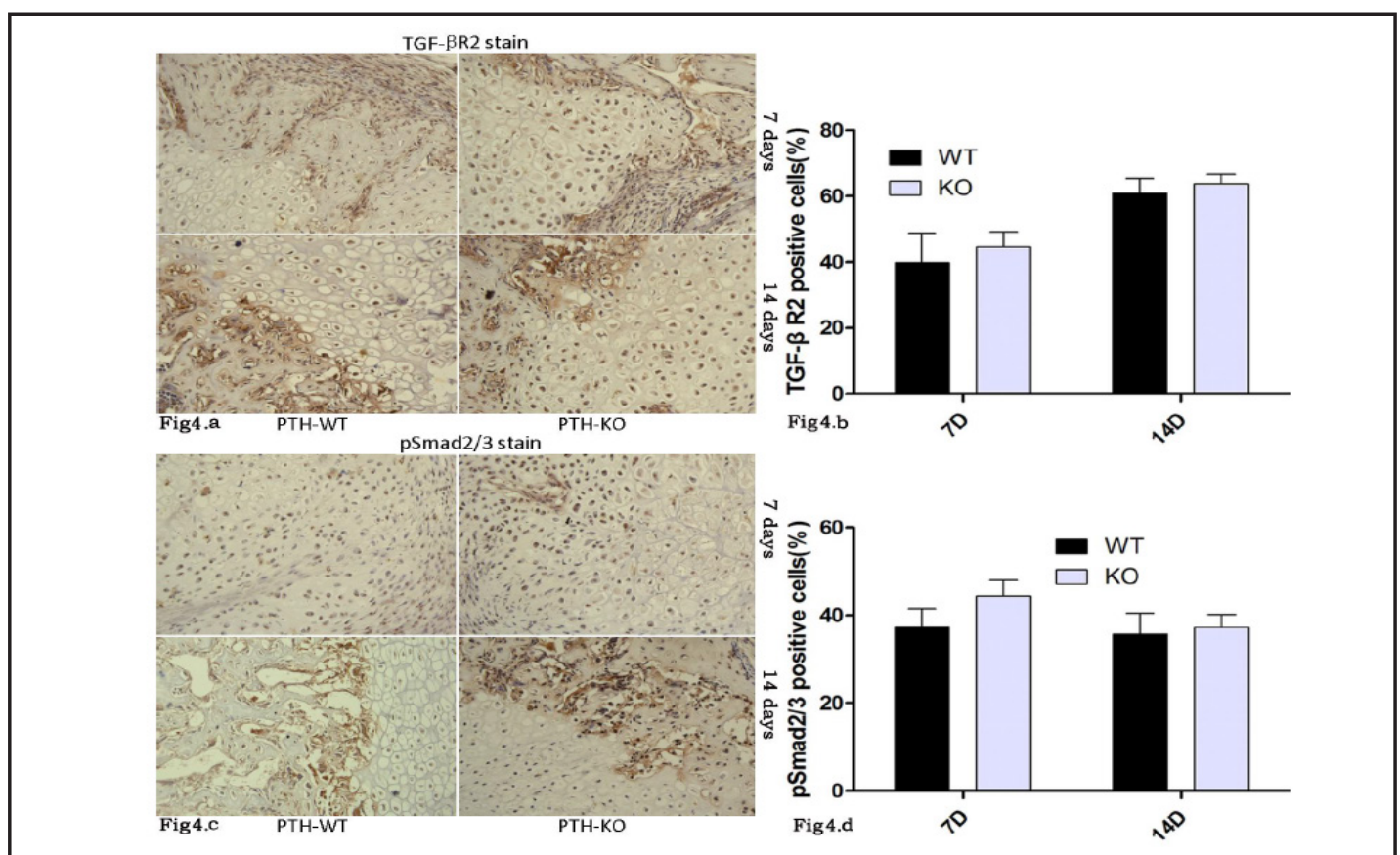

Fig. 4. Endogenous PTH deficiency had no effect in intracellular TGF- $\beta$ signaling pathway. (A) :Immunohistochemical staining of TGF- $\beta$ R2 in the callus of both PTHKO and WT mice at 7 and 14 days after fracture (200x). (B): Comparison of staining intensity of TGF- $\beta$ R2 in callus. (C): Immunohistochemical staining of pSMAD2/3 in the callus of both PTHKO and WT mice at 7 and 14 days after fracture (200x). (D): Comparison of immunohistochemical staining intensity of pSMAD2/3 in callus. ( $*$ : P <0.05 KO mice compared with WT mice).

Fig. 5. PTH gene deletion impaired intracellular signal transduction of BMP pathway. (A) :Immunohistochemical staining of BMPR2 in the callus of both PTHKO and WT mice at 7 and 14 days after fracture (200x). (B): Comparison of staining intensity of BMPR2 in callus $(200 \times)$. (C) :Immunohistochemical staining of pSmad1/5/8 in the callus of both PTHKO and WT mice at 7 and 14 days after fracture (200x). (D): Comparison of staining intensity of pSmad1/5/8 in callus. (*: $\mathrm{P}<0.05 \mathrm{KO}$ mice compared with WT mice).

BMMSCs from PTHKO mice were significantly lower compared to the WT mice $(\mathrm{p}<0.05)$ (Fig. 6C).

Exogenous PTH enhanced ALP activity in
osteoblasts by upregulating BMP signal
transduction through the cAMP-PKA
pathway
Proteins from BMMSCs were extracted Proteins from BMMSCs were extracted at 4 days after osteogenic induction and analyzed by western blot, revealing that PTH knockout reduced intracellular expression of BMPR2, pSMAD1/5/8, and RUNX2. Levels

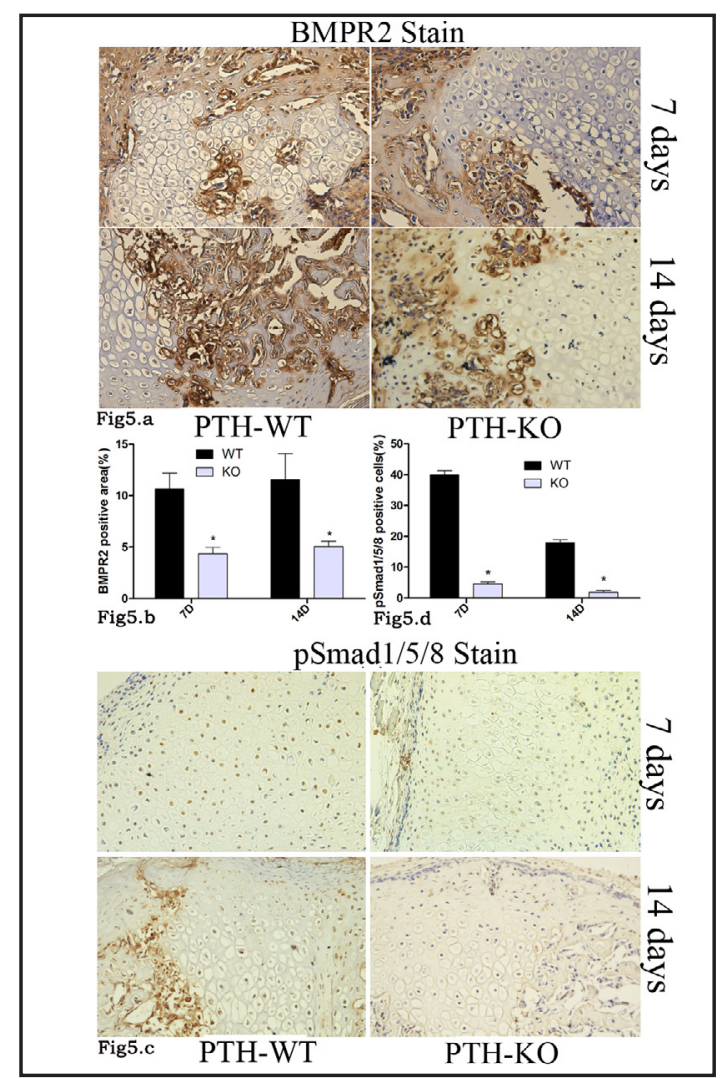




\section{Cellular Physiology Cell Physiol Biochem 2017;42:551-563 \begin{tabular}{l|l|l} 
DOI: 10.1159/000477605 & and Biochemistry & $\begin{array}{l}\text { 2017 The Author(s). Published by S. Karger AG, Basel } \\
\text { www.karger.com/cpb }\end{array}$
\end{tabular}

Fig. 6. PTH gene knockout caused reduced intracellular cAMP levels and decreased CREB expression. (A) : Immunohistochemical staining of pCREB in the callus of both PTHKO and WT mice at 7 and 14 days after fracture (200x). (B): Comparison of staining intensity of pCREB in callus. ${ }^{*}, \mathrm{P}<0.05$ compared with WT mice at the same group. (C): Intracellular cAMP concentration in BMMSCs measured after stimulation of PTH $\left(10^{-7} \mathrm{M}\right)$ for 0 and 30 min. \# ,P<0.05 WT+PTH compared with WT. \&,P $<0.05 \mathrm{KO}+\mathrm{PTH}$ compared with KO. (*: P $<0.05$ KO mice compared with WT mice. \#: $\mathrm{P}<0.05$ WT mice $+\mathrm{PTH}$ compared with WT mice. \&: $\mathrm{P}<0.05$ KO mice +PTH compared with KO mice).
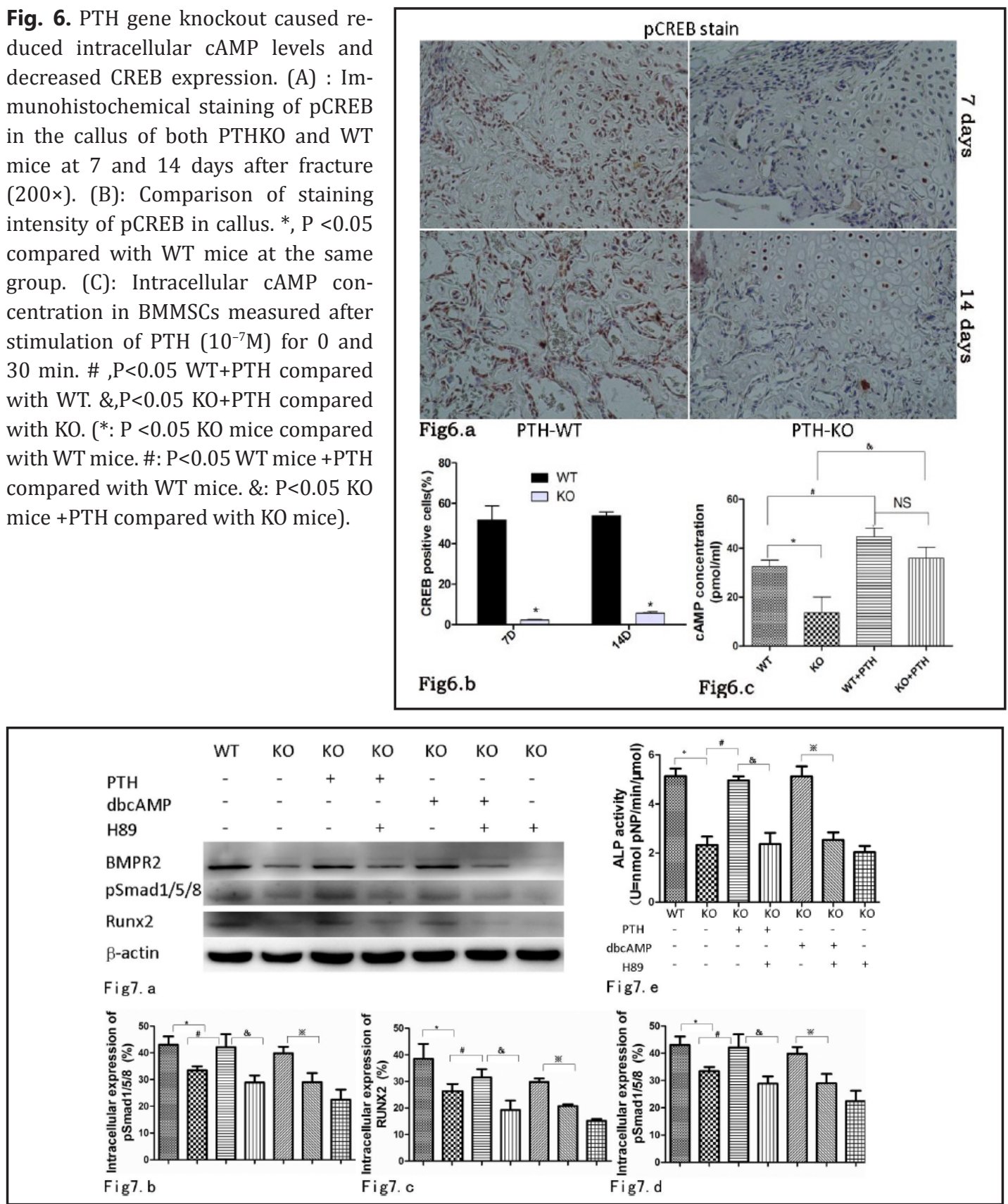

Fig. 7. Exogenous PTH enhanced ALP activity in osteoblasts by up-regulating signal transduction of BMP through cAMP-PKA pathway. (A) Intracellular expression of BMPR2, pSMAD1/5/8 and RUNX2 in BMMSCs at 4 days after osteogenic induction with simulation of PTH, dbcAMP or H89. (B) Comparison of intracellular expression of BMPR2. (C) Comparison of intracellular expression of RUNX2. (D) Comparison of intracellular expression of pSMAD1/5/8. (E) ALP activity in BMMSC-derived osteoblasts at 7 days after induction. (*: P $<0.05$ KO mice compared with WT mice. \#: P<0.05 KO mice + PTH compared with KO mice; \&: P<0.05 KO mice + PTH+H89. $※:$ P<0.05 KO mice +dbcAMP+H89 compared with KO mice +dbcAMP).

of these proteins were increased in the presence of PTH or dbcAMP, while PKA inhibitor (H89) blocked the effects of PTH and dbcAMP on BMPR2 expression (Fig. 7A-C). ALP activity in BMMSC-derived osteoblasts was also measured, showing that ALP activity in the PTHKO group at 7 days after osteogenic induction was significantly lower compared to that observed in the WT group. While exogenous PTH increased ALP activity during osteoblast 


\section{Cellular Physiology Cell Physiol Biochem 2017;42:551-563

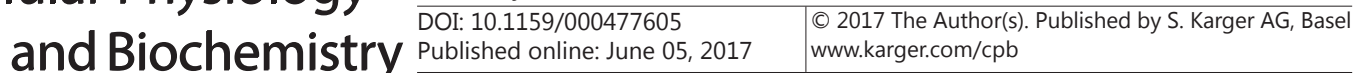 \\ Zhou et al.: Endogenous PTH Enhanced BMPR2 by cAMP/PKA/CREB Pathway}

differentiation of BMMSCs from PTHKO mice, H89 completely blocked PTH and dbcAMP stimulation of ALP activity (Fig. 7D ).

\section{Discussion}

Murine closed-femoral fracture models have been widely used in studies of fracture healing [17, 21, 24]. Femoral fractures are generally produced by blunt trauma, which consistentlyleads to thrypsis. The probability of simple transverse fracture by this method is relatively low, and it is difficult to control the types of fractures and subsequent displacement. Furthermore, it is inappropriate to study different types of fractures as one category due to the fact that they might go through different healing processes. Therefore, an improved openfracture model was adopted in this study $[21,22]$. During the surgery, mouse femoral shafts were cut under direct vision with special scissors to avoid incomplete fracture. Postoperative examinations using X-ray and immunohistochemical staining of callus sections confirmed that all fractures in this study were simple fractures, which subsequently experienced the standard process of secondary fracture healing.

The current study revealed that endogenous PTH deficiency had no impact on chondrocyte differentiation, but rather affected their maturation and hypertrophy. Previous studies have shown that chondrocyte hypertrophy occurred earlier in the PTH-treated callus tissues [25]. Col II is an important component of cartilage matrix. Immunohistochemical staining of the callus identified no significant difference in Col II-positive areas between PTHKO and WT groups at 7-days post-fracture, whereas the positive areas in the PTHKO group were substantially greater than those observed in the WT group at 14-days post-fracture, when cartilage callus in WT mice had already been gradually replaced by woven bone. In contrast, delayed hypertrophy and apoptosis of chondrocytes in PTHKO mice inhibited osteoblasts from entering cartilage matrix, thereby decelerating formation of woven bone and ultimately slowingthe process of fracture healing. Micelles composed of BMMSCs were cultured in vitro in chondrogenic induction medium, and BMMSCs from both PTHKO and WT groups had differentiated into chondrocytes at 12-days post-induction. Furthermore, there was no significant difference in staining intensity between the two groups. However, both in vivo and in vitro experiments confirmed reductions in activity and differentiation capacity of osteoblasts in PTHKO mice, indicating strong effects of PTH knockout on osteoblasts. Coll is the major component of collagen fibers secreted by osteoblasts [18]. Immunohistochemical analysis showed that staining intensity of Col I callus decreasedsignificantly in PTHKO mice. ALP is a marker of osteoblast differentiation [26], and ALP staining and activity assays of BMMSC-derived osteoblasts revealed reductions in both osteoblastic differentiation capacity and ALP activity of MSCs from PTHKO mice, suggesting that endogenous PTH might somehow accelerate fracture healing by increasing osteoblast differentiation and activity. Additionally, PTH might also speed fracture healing by promoting endochondral ossification through stimulation of chondrocyte hypertrophy and apoptosis.

Differentiation and maturation of chondrocytes and osteoblasts are curial to the process of bone-fracture healing. Local hypoxia after fracture can cause BMMSC to differentiate. And hypoxia was able to increase ALP activity and osteoblast mineralization [27]. During the late stages of endochondral bone formation, chondrocytes undergo hypertrophy, followed by apoptosis, and form lacunae in cartilage matrix. Osteoblasts enter lacunae along blood vessels attached to the surface of the remnants of cartilage matrix and secrete collagen and bone matrix, which gradually replaces the cartilage matrix [28].

SOX9 is a critical growth factor for cartilage formation [29] by stimulating differentiation of MSCs into chondrocytes and preventing chondrocyte hypertrophy [12]. RUNX2 is a key transcription factor regulating osteoblast differentiation and maturation, which activates osteoblastic differentiation of MSCs and regulates maturation of osteoblasts, intramembranous ossification, as well as endochondral ossification [30]. Additionally, RUNX2 can stimulate chondrocytehypertrophy and apoptosis and promote endochondral 
ossification [12]. Our results showed that RUNX2 expression in the callus of PTHKO mice was significantly lower compared to WT mice, whereas SOX9 was expressed at the same level in both groups, suggesting that PTH mainly affected endochondral bone formation by RUNX2.

TGF- $\beta$ s/BMPs play essential roles in regulating osteoblast and chondrocyte growth, maturation, and apoptosis. TGF- $\beta$-/BMP-signaling molecules act on type I and type II serine/ threonine kinase receptor complexes in target-cell membranes. Ligand binding to the type II kinase receptor (TGF- $\beta$ R2 and BMPR2) stimulates the intrinsic kinase activity of the receptor, which subsequently phosphorylates and activates the type I receptor. Activated type I receptor phosphorylates receptor-dependent SMAD proteins (R-SMAD, namely SMAD1/2/3/5/8). R-SMADs aggregate and form heterodimeric complexes with CoSMAD proteins (namely SMAD4) and translocate into the nucleus, where they cooperatively regulate the transcription of target genes with other transcription factors. Additionally RUNX2 is the last transcription factor in the TGF- $\beta$-/BMP-signaling pathway [30]. In this study, PTHKO mice displayed reduced expression of BMPR2 and pSMAD1/5/8, but not of TGF- $\beta$ R2 and pSMAD2/3 as compared to WT mice, indicating the endogenous PTH deficiency inhibited signal transduction in the BMP pathway, but had no significant effect on TGF- $\beta$ signal transduction.

PTH treatment resulted in CREB phosphorylation on the activating serine 133 residue [31]. The effects of PTH are primarily mediated by activating the cAMP/PKA signaling pathway through binding to G-protein-coupled receptors in target-cell membranes [32]. PKA is composed of two regulatory and two catalytic subunits, and is activated as a cAMP molecule binds to the two regulatory subunits and releases the two catalytic subunits, which enter the cell nucleus and activate gene expression by phosphorylating Ser133 of CREB [33]. The cAMP/PKA/CREB signaling pathway can stimulate the BMP signaling pathway [34, 35], as well as expression of markers related to bone formation, such as bone sialoprotein and osteocalcin [36].

Results in this study showed that PTH knockout resulted in decreased intracellular cAMP levels and inhibited signal transduction of the cAMP/PKA/CREB pathway in mice. Immunohistochemical staining illustrated a significantly lower staining intensity of pCREB in the callus of PTHKO mice compared to WT mice. Furthermore, western blotconfirmed that BMPR2 expression was significantly decreased during osteoblastic differentiation of BMMSCs from PTHKO mice. While BMPR2 expression was upregulated following stimulation of dbcAMP, it was significantly downregulated after administration of PKA inhibitor H89, which inhibited the activity of the cAMP/PKA/CREB pathway. The results of assessing ALP activity at 7 days after osteogenic induction were also consistent with our earlier findings. Therefore, PTH knockout caused reduced signal transduction in the cAMP/PKA/CREB pathway andinhibited the BMP signaling pathway by affecting BMPR2 expression.

Additionally, this study showed that PTH knockout had no effect on the TGF- $\beta$ signaling pathway. However, this does not imply that no interaction exists between the TGF- $\beta$ and PTH pathways. Studies showed that binding of PTH to PTHR1 can stimulate TGF- $\beta$ R2 to form complexes with and phosphorylate PTHR1, which simultaneously diminishes the effects of TGF- $\beta$ and PTH on bone development $[37,38]$.

In summary, this study revealed that endogenous PTH increased intracellular cAMP concentrations and improved BMPR2 expression through the cAMP/PKA/CREB pathway, thereby stimulatingRUNX2expression by enhancing signal transduction in the BMP/ pSmad1/5/8 pathway. RUNX2 further expedited endochondral bone formation by promoting chondrocyte hypertrophy and apoptosis, stimulated differentiation and maturation of osteoblasts, and ultimately accelerated the process of fracture healing.

\section{Acknowledgements}

This work was supported by the funds from National Natural Science Foundation of China (No.81401800). 


\section{Cellular Physiology Cell Physiol Biochem 2017;42:551-563 \begin{tabular}{l|l} 
DOI: 10.1159/000477605 & $\begin{array}{l}\text { O 2017 The Author(s). Published by S. Karger AG, Basel } \\
\text { www.karger.com/cpb }\end{array}$
\end{tabular} Zhou et al.: Endogenous PTH Enhanced BMPR2 by cAMP/PKA/CREB Pathway}

\section{Disclosure Statement}

No conflict of interest to declare.

\section{References}

1 Deschaseaux F, Sensebe L, Heymann D: Mechanisms of bone repair and regeneration. Trends Mol Med 2009;15:417-429.

-2 Soltanoff CS, Yang S, Chen W, Li YP: Signaling networks that control the lineage commitment and differentiation of bone cells. Crit Rev Eukaryot Gene Expr 2009;19:1-46.

-3 Huang W, Yang S, Shao J, Li YP: Signaling and transcriptional regulation in osteoblast commitment and differentiation. Front Biosci 2007;12:3068-3092.

4 Tomlinson RE, McKenzie JA, Schmieder AH, Wohl GR, Lanza GM, Silva MJ: Angiogenesis is required for stress fracture healing in rats. Bone 2013;52:212-219.

-5 Cleary MA, van Osch GJ, Brama PA, Hellingman CA, Narcisi R: FGF, TGFbeta and Wnt crosstalk: Embryonic to in vitro cartilage development from mesenchymal stem cells. J Tissue Eng Regen Med 2015;9:332-342. Guo X, Wang XF: Signaling cross-talk between TGF-beta/BMP and other pathways. Cell Res 2009;19:71-88. Worster AA, Brower-Toland BD, Fortier LA, Bent SJ, Williams J, Nixon AJ: Chondrocytic differentiation of mesenchymal stem cells sequentially exposed to transforming growth factor-beta1 in monolayer and insulin-like growth factor-I in a three-dimensional matrix. J Orthop Res 2001;19:738-749.

-8 Yang W, Guo D, Harris MA, Cui Y, Gluhak-Heinrich J, Wu J, Chen XD, Skinner C, Nyman JS, Edwards JR, Mundy GR, Lichtler A, Kream BE, Rowe DW, Kalajzic I, David V, Quarles DL, Villareal D, Scott G, Ray M, Liu S, Martin JF, Mishina Y, Harris SE: Bmp2 in osteoblasts of periosteum and trabecular bone links bone formation to vascularization and mesenchymal stem cells. J Cell Sci 2013;126:4085-4098.

$\checkmark 9$ Dean DB, Watson JT, Jin W, Peters C, Enders JT, Chen A, Moed BR, Zhang Z: Distinct functionalities of bone morphogenetic protein antagonists during fracture healing in mice. J Anat 2010;216:625-630.

10 Wagner DO, Sieber C, Bhushan R, Borgermann JH, Graf D, Knaus P: BMPs: From bone to body morphogenetic proteins. Sci Signal 2010;3:r1.

11 Yi JJ, Barnes AP, Hand R, Polleux F, Ehlers MD: TGF-beta signaling specifies axons during brain development. Cell 2010;142:144-157.

12 Zelzer E, Olsen BR: The genetic basis for skeletal diseases. Nature 2003;423:343-348.

13 Aspenberg P: Annotation: Parathyroid hormone and fracture healing. Acta Orthop 2013;84:4-6.

14 Migliore A, Broccoli S, Massafra U, Bizzi E, Frediani B: Mixed-treatment comparison of anabolic (teriparatide and PTH 1-84) therapies in women with severe osteoporosis. Curr Med Res Opin 2012;28:467-473.

15 Yamamoto T, Hamaya E, Sowa H: [Evidence for osteoporosis treatment using PTH (1-34) daily subcutaneous injection]. Clin Calcium 2012;22:399-406.

16 Madore GR, Sherman PJ, Lane JM: Parathyroid hormone. J Am Acad Orthop Surg 2004;12:67-71.

17 Jilka RL: Molecular and cellular mechanisms of the anabolic effect of intermittent PTH. Bone 2007;40:1434-1446.

-18 Ren Y, Liu B, Feng Y, Shu L, Cao X, Karaplis A, Goltzman D, Miao D: Endogenous PTH deficiency impairs fracture healing and impedes the fracture-healing efficacy of exogenous PTH(1-34). Plos One 2011;6:e23060.

19 Lin EA, Liu CJ, Monroy A, Khurana S, Egol KA: Prevention of atrophic nonunion by the systemic administration of parathyroid hormone (PTH 1-34) in an experimental animal model. J Orthop Trauma 2012;26:719-723.

20 Kim JM, Choi JS, Kim YH, Jin SH, Lim S, Jang HJ, Kim KT, Ryu SH, Suh PG: An activator of the cAMP/PKA/ CREB pathway promotes osteogenesis from human mesenchymal stem cells. J Cell Physiol 2013;228:617626.

21 Doyon AR, Ferries IK, Li J: Glucocorticoid attenuates the anabolic effects of parathyroid hormone on fracture repair. Calcif Tissue Int 2010;87:68-76. 


\section{Cellular Physiology Cell Physiol Biochem 2017;42:551-563 \begin{tabular}{l|l} 
DOI: 10.1159/000477605 & $\begin{array}{l}\text { O 2017 The Author(s). Published by S. Karger AG, Basel } \\
\text { www.karger.com/cpb }\end{array}$
\end{tabular} \\ Zhou et al.: Endogenous PTH Enhanced BMPR2 by cAMP/PKA/CREB Pathway}

22 Yuan L, Lin Z, Fu Z, Meng Y, Huang Z, Wu X, Yang D, Jiang J: [Effects of signaling-selective parathyroid hormone peptide analog on fracture healing in orchiectomized mouse models]. Nan Fang Yi Ke Da Xue Xue Bao 2013;33:182-187.

23 Nakao Y, Koike T, Ohta Y, Manaka T, Imai Y, Takaoka K: Parathyroid hormone enhances bone morphogenetic protein activity by increasing intracellular 3', 5'-cyclic adenosine monophosphate accumulation in osteoblastic MC3T3-E1 cells. Bone 2009;44:872-877.

24 Takahata M, Awad HA, O'Keefe RJ, Bukata SV, Schwarz EM: Endogenous tissue engineering: PTH therapy for skeletal repair. Cell Tissue Res 2012;347:545-552.

-25 Kakar S, Einhorn TA, Vora S, Miara LJ, Hon G, Wigner NA, Toben D, Jacobsen KA, Al-Sebaei MO, Song M, Trackman PC, Morgan EF, Gerstenfeld LC, Barnes GL: Enhanced chondrogenesis and Wnt signaling in PTHtreated fractures. J Bone Miner Res 2007;22:1903-1912.

-26 Sugawara Y, Suzuki K, Koshikawa M, Ando M, Iida J: Necessity of enzymatic activity of alkaline phosphatase for mineralization of osteoblastic cells. Jpn J Pharmacol 2002;88:262-269.

27 Huang J, Peng J, Cao G, Lu S, Liu L, Li Z, Zhou M, Feng M, Shen H: Hypoxia-Induced MicroRNA-429 promotes differentiation of MC3T3-E1 osteoblastic cells by mediating ZFPM2 expression. Cell Physiol Biochem 2016;39:1177-1186.

28 Buckwalter JA, Glimcher MJ, Cooper RR, Recker R: Bone biology. II: Formation, form, modeling, remodeling, and regulation of cell function. Instr Course Lect 1996;45:387-399.

29 Yang B, Guo H, Zhang Y, Chen L, Ying D, Dong S: MicroRNA-145 regulates chondrogenic differentiation of mesenchymal stem cells by targeting Sox9. Plos One 2011;6:e21679.

Z30 Zaidi M: Skeletal remodeling in health and disease. Nat Med 2007;13:791-801.

-31 Schnoke M, Midura RJ: Pulsed electromagnetic fields rapidly modulate intracellular signaling events in osteoblastic cells: Comparison to parathyroid hormone and insulin. J Orthop Res 2007;25:933-940.

-32 Yang D, Guo J, Divieti P, Bringhurst FR: Parathyroid hormone activates PKC-delta and regulates osteoblastic differentiation via a PLC-independent pathway. Bone 2006;38:485-496.

-33 Bidwell P, Joh K, Leaver HA, Rizzo MT: Prostaglandin E2 activates cAMP response element-binding protein in glioma cells via a signaling pathway involving PKA-dependent inhibition of ERK. Prostaglandins Other Lipid Mediat 2010;91:18-29.

-34 Zhang R, Edwards JR, Ko SY, Dong S, Liu H, Oyajobi BO, Papasian C, Deng HW, Zhao M: Transcriptional regulation of BMP2 expression by the PTH-CREB signaling pathway in osteoblasts. Plos One 2011;6:e20780.

-35 Ionescu AM, Drissi H, Schwarz EM, Kato M, Puzas JE, McCance DJ, Rosier RN, Zuscik MJ, O'Keefe RJ: CREB Cooperates with BMP-stimulated Smad signaling to enhance transcription of the Smad6 promoter. J Cell Physiol 2004;198:428-440.

-36 Matsuo N, Tanaka S, Gordon MK, Koch M, Yoshioka H, Ramirez F: CREB-AP1 protein complexes regulate transcription of the collagen XXIV gene (Col24a1) in osteoblasts. J Biol Chem 2006;281:5445-5452. Atfi A, Baron R: PTH battles TGF-beta in bone. Nat Cell Biol 2010;12:205-207.

Qiu T, Wu X, Zhang F, Clemens TL, Wan M, Cao X: TGF-beta type II receptor phosphorylates PTH receptor to integrate bone remodelling signalling. Nat Cell Biol 2010;12:224-234. 